$$
2 i-42 y
$$

\title{
LA-6412-MS
}

informal Report

UC-20

Reporting Date: August 1976

Issued: August 1976

\section{Plasma End-Loss Studies on Síylla I-C}

\author{
by
}

K. F. McKenna

T. M. York*

"Visiting Staff Member. Pennsylvania State University, University Park, PA 16801.

An Allirmative Action/Equal Opportunity Employer 
Printed in the United States of America. Available from National Technical Information Service

U.S. Department of Commerce

5285 Port Roysl Road

Syringfield, VA 22161

Price: Printed Copy \$4.00 Microfiche \$2.25

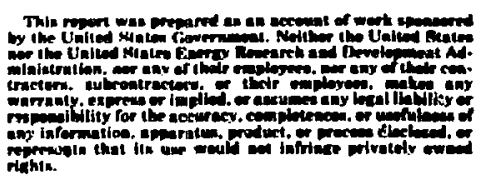




\begin{abstract}
The end-loss process in the collision dominated Scylla I-C plasma has been irvestigated with a local pressure sensitive diagnostic, integrated density messurements and oxially arrayed diamagnetic loop probes. The development of a plasma loss orifice, well within the theta-pinch coll, has been identified. The magnitude of the observed orifice is round to be in excellent agreement with that predicted from collisional MFD theories. The axially flowing plasma is well confined until it flows through the los: orifice. After passing through the oriffee, rapid axial expansion is observed. An indication of the existence of invard traveling rarefaction waves has been observed from the plasma midplane temperature data; an abrupt decrease in the plasma temperature at $t=6.5 \mathrm{\mu s}$ corresponds to the predicted time of arrival of rarefaction waves at the coll midplane. The plasma loss rate derived from the pressure data indicates an initial period (t<h $\mu s$ ) of rapia particle loss followed by a period ( $t>i_{1}$ us) of gradual decay in the loss rate. This intial period of high loss rate is predicted from the MHD flow theories when the measured, time dependent plasma paraneters are substituted into the analytical models. The lose rate determined from the end-on interferogrars toes not respond to the deteiled structure of the plasma loss process.
\end{abstract}

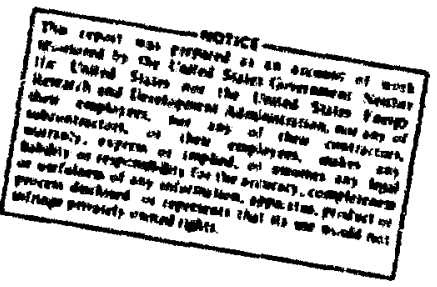




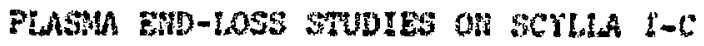

in

K. F. Wenenns and $T$, W. Work

\section{INTRODUCTTON}

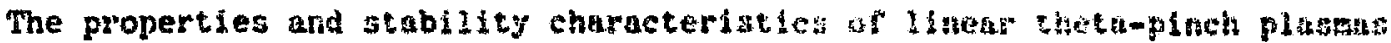

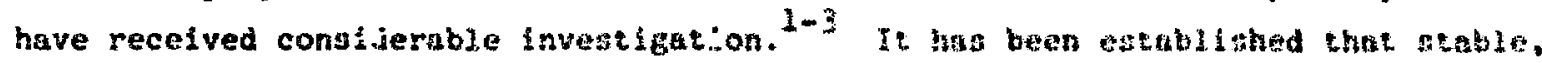
high energy plasmas of fusion suterest can be produced in theste devices. Houevor,

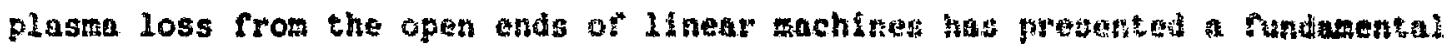

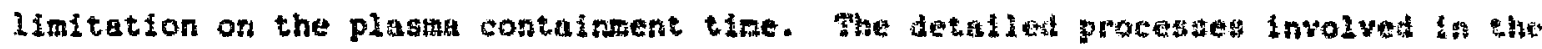

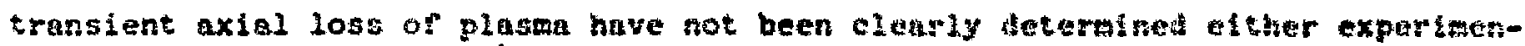

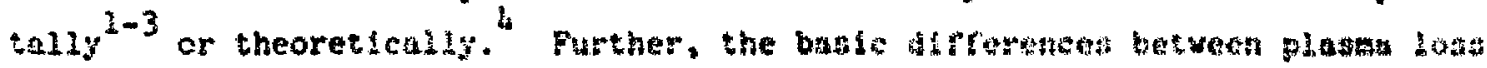

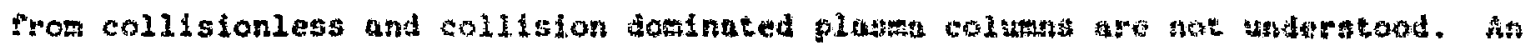

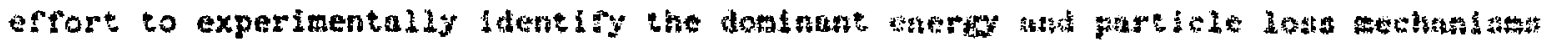

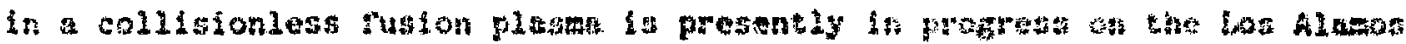
Scylik IU-P thets ptnch.

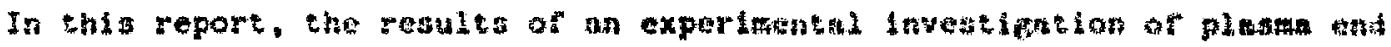

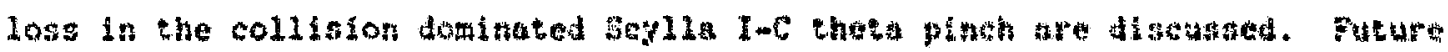

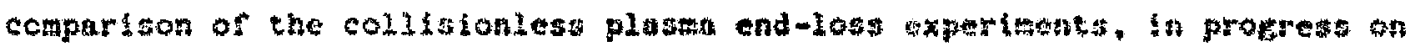

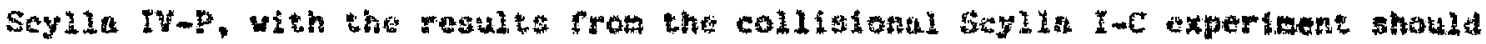

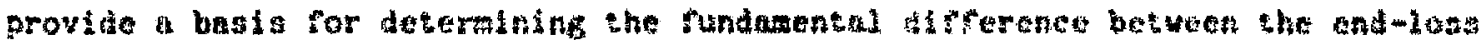
processeg in these two pinstar regimes.

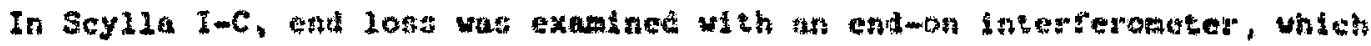

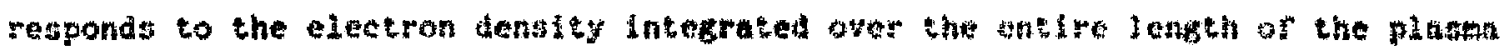

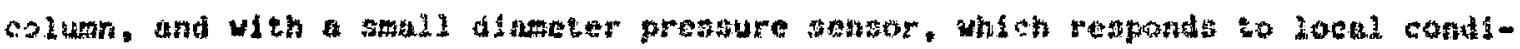

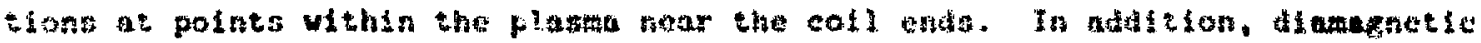

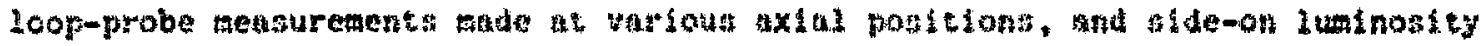

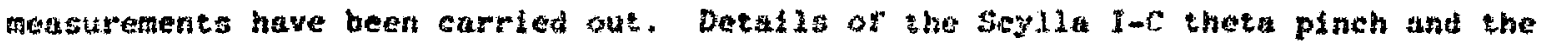
plasma parameters, over a wate range of intelad mil pressures, have been 3 iscussed in eompanion report. 
II. PLASMA END LOSS; EXPERIMENTAL AND THEORETICAL CONSIDERATIONS

The escape of plasma from the ends of high-B linear theta-pinch devices can be characterized by the dimensions of the loss orifice (often referred to as a "hole" or "throat" region) assumed to be located at the ends of the theta-pinch plasma column. The mechanisms determining the size of the orifice, its spatial and temporal evolution, and the transport of energy and particles througli the orifice have not been identified. It cannot be assumed that the properties of the confined source plasma define the characteristics of the loss orifife since the orifice can be in direct communication with the external environment through the magnetic field lines which connect to it. Specifically, if the inferaction between the diverging field lines and vacuum vessel walls at the end of the theta pinch is strong, then the end-loss process may well be dominated by the properties of the discharee tube walls. Cold plasma, resulting from the preionization discharge as well as neutral gas external to the main plasma column, may also provide a mechanism for altering the structure of the loss orifice. The fact that end effects can significantly influence the stability characteristics of iinear plasma systems has clearly been established. 2,3

\section{A. Sheath Models of the Loss Orifice}

Considering only the properties of the internal source plasma, the dimensions of the loss orifice would be expected to be on the order of the plasma sheath thickness. The sheath arises as a result of plasma penetration into the confining uagnetic field. The minimur sheath thickness cen be estimated from a consideration of the thinnest possible boundary separating a collisionless $B=1$ $(B$ is the ratio of plasma pressure to confining magnetic field pressure) plasma from the external magnetic field. Grad ${ }^{7}$ has treated the steady one-dimensional transition zone between a collisionless fleld-free Maxwellian plasma at $x=-\infty$ and a uniform magnetic field $B_{2}$ at $x=+\infty$. In this analysis all particles are or equal mass ( $\left.m-m_{e}-m_{i}\right)$ and particle "trapping" within the sheath is not allowed. In Gra's solution for the thinnest possible sheath, the plasma is found to extend asymptotically intc the field with a scale length $\lambda$, while the field decays to zero at a finite distance within the plasma. The leneth scale $\lambda$ can be idertified as the particle cyro radius composed of the magnetic field at $x=-\infty$ and the mean thermal speed $v_{0}$ at $x=+\infty$, 


$$
\lambda=\frac{\mathrm{mu}_{\mathrm{o}}^{\mathrm{L}}}{\mathrm{eB}_{\mathrm{z}}}
$$

Extending the solution for the transition layer to the more physical case were the electrons and ions have unequal masses $\left(m_{i}>m_{e}\right)$ requires the inclusion of the space charge electric field $E_{x}(x)$ developed perpendicular to $B_{z}(x)$. Restricting the velocity of the electrons and ions to a single value $v_{0}(\infty)$ and again no: permitting trapped particles, the transition layer in this case is shown ${ }^{8}$ to be chaiacterized by a length scale $\lambda$ 'equal to the geometric mean of the electron and ion gyro-radii,

$$
\lambda^{\prime}=\left(m_{i} m_{e}\right)^{1 / 2} \frac{v_{0} c}{e B_{z}} .
$$

The further extension of the transition layer problem tc include an isotropic plasma velocity distribution at - $\infty$ does not yield a self-consistent solution unless trapped electrons, in the sheuth, are permitted. However, non-unique solutions are then obtained due to the arbitrariness of the trapped electron distribution. Morse ${ }^{9}$ has reduced this arbitrariness by considering the temporal development of the transition zone in which electrons are trapped in a predictable, but non-unique, feshion in the presence of is slowly rising magnetic fielä. Morse's solution, for a Maxwellian plasma of arbitrary electron and ion temperatures, predicts $a$ sheath thickness having a scale length $\lambda^{\prime \prime}$ on the order of the electron Byro-radii,

$$
\lambda^{\prime \prime}=\frac{m_{e_{0}^{v_{0}}}^{v_{0}}}{e B_{2}} .
$$

According to the collisionless theoretical treatments discussed above, the radius of the loss orifice would be expected to have a value between the electror gyro-radius and the geometric mean of the electron and ion gyro-radii, based on the internal plaswa properties and the existence of a radial space charge electric field perpendicular to the confining magnetic field. Considering end effects, if an electron source can be generated outside the plasma column but in contact with the confining magnetic fields, then the radial electric field wiihin the plasma 
culumn sheath can be short-circuited by electrons flowing along the magnetic field lines. In this case the ions can penetrate the sheath to a deptr of an ion gyroradius, increasing the size of the loss orifice and, correspondingly, the magnitude of the particle loss, by un order of magnitude. End-shorting has been observed in linear theta pinches ${ }^{10,11}$ and is suspected as the cause of the plasma "wobble" instability; this instability results when the plasma ions are forced to carry their own diamagnetic current which prodices a net plasma rotation. 2,3

For the case where the space charge electric field has been short-circuited by end effects, an alternative expression for the losis orifice radius can be de-ived from a consideration of flux conservation in the plasma sheath. ${ }^{12}$ The magnetic flux contained within a plasma sheath of thickness $r_{i}\left(r_{i}=\right.$ ion gryoradius), surrounding a collisionless $B=1$ plasma column of radius $r$ can be shown to be $\Phi=2 \pi B_{0} r_{p} r_{i}$ for $B_{z}(r)=0$ at $r=r_{p}-r_{i} / 2$ and $B_{2}(r)=B_{0}$ at $r=$ $r_{p}+r_{i} / 2$ where $B_{0}$ is the external magnetic field. Assuming that the transverse plasma pressure drops to zero in the loss orifice then $\phi=B_{0} \pi r_{0}^{2}$ where $r_{0}$ is the loss orifice radius. Accordingly, for $\phi=$ constant,

$$
r_{0}=\sqrt{2}\left(r_{p} r_{i}\right)^{1 / 2}
$$

i.e., flux conservation within the sheath, in the absence of radial electric fields, predicts a loss orifice with a radius equal, within a numerical constant, to the geometric mean of the plasma and ion gyro-radii.

B. Trapped Flux Models of the Loss Orifice

Collisional MHD theories predict that the size of the loss orifice is determined by the magnitude of the magnetic fields which pass through it. Taylor and Wesson ${ }^{13}$ have treated the case of steady plasma flow out the ends of a linear theta pinch. Their model predicts a loss orifice radius given by

$$
r_{0}=r_{p}(1-\beta)^{1 / 4}
$$

where $r_{p}$ and $B$ are evaluated for the source plasma the coil midplarie. Wesson 14 has considered the unsteady collisichal flow case in which rarefaction waves propagate in from the endis of the plasma column. In Wesson's model the mimimum plasma radius is given by 


$$
r_{0}=r_{p}\left(\frac{1-B}{Y}\right)^{1 / 6}
$$

where $\gamma$ is the ratio of specific heats and $r_{p}$ and $B$ are again evaluated for the source plasma.

The above theoretical treatments su'fer from the unrealistic prediction tinat the loss orifice and, this, the particle loss rate go to zero as $B$ goes to uniiy. However, experimental evidence ${ }^{15}$ does exist which indicates that Wesson's 14 unsteady flow theory exhibits the essential physical features observed in collisional plasmas.

\section{Energy Loss Mechanisms}

Neglecting radiation, the axial loss of energy from a theta-pinch plasma column can be as limiting, and possibly more limiting, to the plasma containment time as particle loss. As in the case of particle loss, it cannot be assumed that the properties of the source plasma define the characteristics of the energy loss process. Specifically, the axial transport of particle energy by thermal conduction requires the existence of a temperature gradient between the source plaska and the external boundary. The temperature gradient will be maintained if the energy sink at the boundary can dissipate, at a sufficient rate, all the energy incident upon it. If the sirk is inadequate, for example a total vacuum region, then a temperature gradient cannot be maintained and the plasmu energy can unly be lost through particle transpert. However, in most theta-pinch configurations, an adequate energy sink is provided by the discharge tube walls at the ends of the theta pinch.

Considering the dominant energy loss mechanisms to be diffusive electron heat conduction and axial electron and ion mass transport, an estimate of the relative importance of these two processes can be made from kinetic theory. 16,17 The diffusive axial electron energy flux $Q_{e}$ can be expressed as

$$
q_{e}=\frac{n_{e}^{v} e}{4} 2 k \lambda \text { ee } \frac{d T e}{d z} \approx n_{e} v_{e} k T e\left(\frac{\lambda_{e e}}{\ell}\right) \text {, }
$$

where $\lambda_{e e}\left(=\lambda_{i j}\right.$ for $\left.T_{e}=T_{i}\right)$ is the electron-electron mean free path, $v_{e}$ is the electron thermal velocity and $l$ is the plasma column length. The rate of change of the total electron energy $W_{e}$, per unit length, is given by 


$$
\frac{d w_{e}}{d t}=\frac{3}{2} \ln _{e^{k}} \frac{d T_{e}}{d t}
$$

The characteristie time $\tau_{c}$ for axisl energy transport by thermal conduction is olbtained by equating $Q_{e}$ and $d W_{e} / d t$, which yields

$$
\tau_{c} \sim \frac{\ell}{v_{e}} \cdot \frac{\ell}{\lambda_{e e}} .
$$

The characteristic time $\tau$ for energy loss by particle transport is given by

$$
\tau \sim \frac{\ell}{v_{i}}
$$

where $v_{i}$ is the ion thermal velocity. Accordingiy, thermal diffusion is the dominant energy loss mechanism if

$$
\frac{\ell}{v_{i}}>\frac{\ell}{v_{e}} \cdot \frac{\ell}{\lambda_{e e}}
$$

or rewritten,

$$
\frac{\lambda}{\ell}>\frac{v_{i}}{v_{e}}=\left(\frac{m_{e} T_{i}}{m_{i} T_{e}}\right)^{1 / 2}
$$

For equal ion and electron energies $\mathrm{T}_{e}=\mathrm{T}_{i}$ so that $v_{i} / v_{e}=\left(\mathrm{m}_{e} / \mathrm{m}_{i}\right)^{1 / 2}=0.017$. For the parameters of the Scylla I-C plasma $\lambda_{e e} / \ell \approx 0.001$, and the energy loss process is therefore dominated by particle end loss. However, in higher energy theta pinchis, such as Scylla IV-P, $\lambda_{\text {ee }} / \ell$ will exceed the limiting value of 0.017 and plasma energy loss should then be dominated by thermel diffusion. It should be noted that the simple analysis presented above loses validity as $\lambda_{\text {ee }}$ approaches $\ell$ since the diffusion convept becomes less applicable as the plasma becomes more collisionless. 
III. THE SCYLLA I-C THETA PINCH

Seylla $I-C^{6}$ stores 78 kJ at a primary bank voltage of $40 \mathrm{kV}$. Fifty-four 1.8-wF capacitors read the $300-\mathrm{cm}$ long, 10.5-crn diameter single-turn compression coij. The initial $E_{\theta}$ at the inner well of the $3.6-\mathrm{cm} i . d$. quartz discharge tube is $0.26 \mathrm{kV} / \mathrm{cm}$ and a peak crowbarred compression field of $33 \mathrm{~kJ}$ is obtained $2.0 \mathrm{\mu s}$ after discharge lnitlation. Gas preionization is accomplished with an axial z-pinch discharge. The plesma end-loss experiments were carried out with a thetepinch fill pressure of 100 mtorr deuteriun.

IY. PROPERTIES OF THE THETA-PINCH SOURCE PLASMA

A. Plagma Luminosicy Measurements

Stde-on streak photographs of the source plasma were taken at the coil midplane. The initial implosion phase, a subsequent period of radial oscillations or about 1.3-ws duration, and a following highly stable and reproducible quiescent plasma colum phase were observed. ${ }^{6}$ The radial oscillation can be attributed to magnetic field dffrusion and trapping during the implosion phase. 6 The plasma colum 11 fetiwe is terrinated at $t>8.5$ us by the m=2 instability. B. Source Piasma Density Measurements

The evolution of the source plasma density distribution was determined using a 30-ns pulsed ruby laser interferometer in an end-on configuration. From examination of the interferograms, Fig. 1, the plasma column formation phase, $t<1.0 \mathrm{\mu s}$, is found to be dominated by high m-number $(\mathrm{m}>6)$ flutes. At later times, $t>1.5 \mathrm{\mu s}$, the rlutes disappear and a well confined high-density plasma column was observed for several microseconds.

Electron density profiles reduced from the end-on interferograms, for an assumed column length of $100 \mathrm{~cm}$, are shoun in Fig. 2. The density profiles exhibit a lang toll for $t \geq 3 \mathrm{us}$, which may be a result of plasmg flow from the ends of the column. An average peak density on axis, $n_{A}$. of approximately $1.1 \times 10^{17} \mathrm{~cm}^{-3}$ is obtained at meximum compression, $t=2.0 \mu \mathrm{s}$. The on-axis density decays to a value of about $0.3 \times 10^{17} \mathrm{~cm}^{-3}$ just prior to column filementation; a slight increase in on-axis density between 5 and $6 \mu \mathrm{s}$ results from the low frequency oscillation of the main compression field. A $1 /$ e plasma radius, $r_{p}$, of $\sim 0.6 \mathrm{~cm}$ is indicated from the density profiles.

c. Plasma Excluded Flux Messurements

The effective radius, $r_{\in f}$, at which magnetic flux is excluded from the compressed plasma was determined from diamagneicic loop-probe measurements. 6 


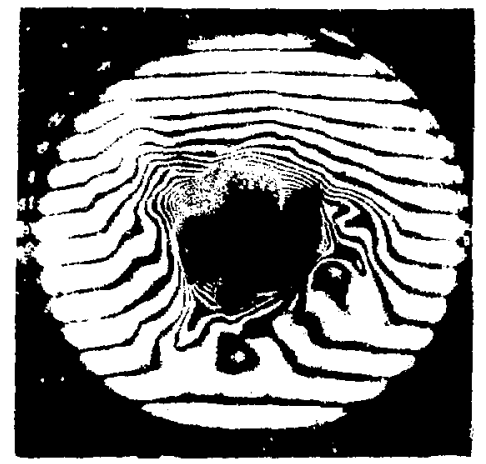

$1.0 \mu \mathrm{sec}$

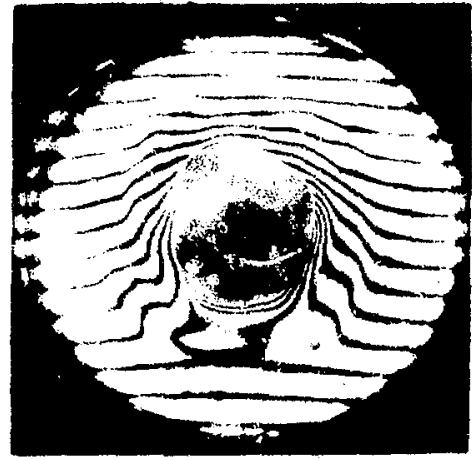

\section{$2.0 \mu$ sec}

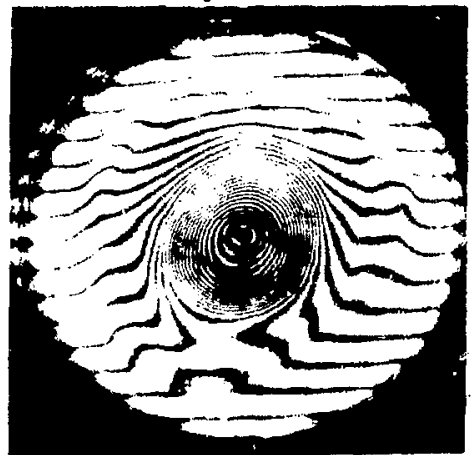

$3.0 \mu \mathrm{sec}$

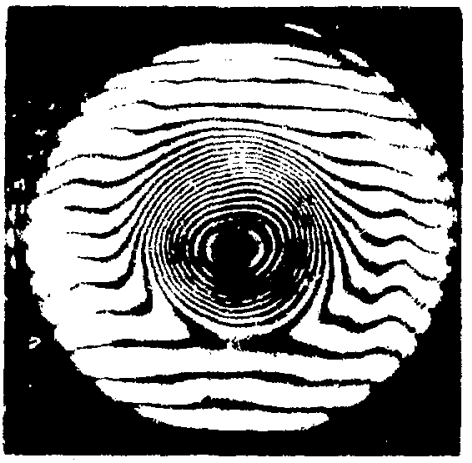

$4.0 \mu \mathrm{sec}$

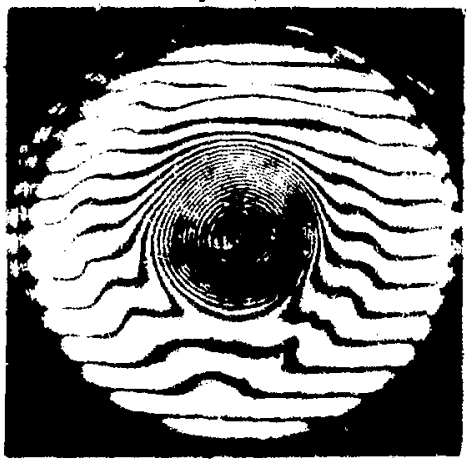

$5.0 \mu \mathrm{sec}$

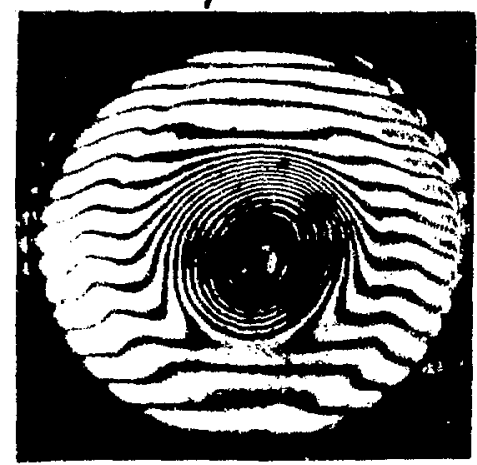

$6.3 \mu \mathrm{sec}$

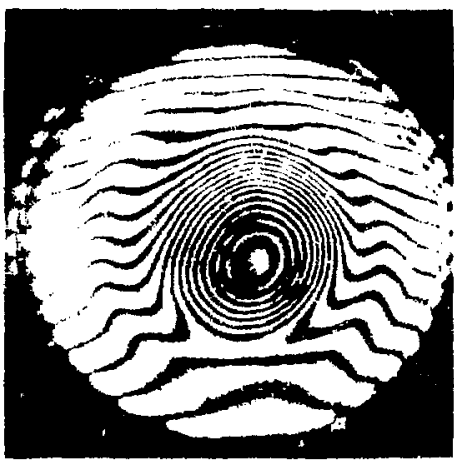

$7.3 \mu$ sec

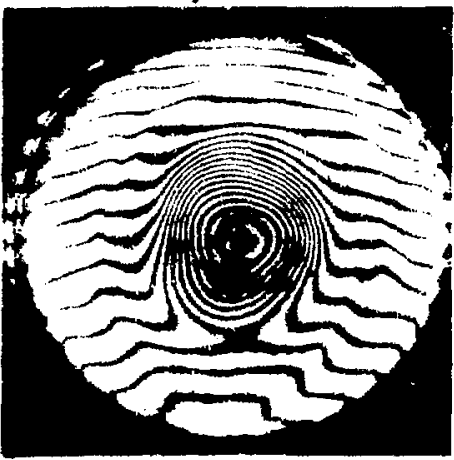

$8.0 \mu \mathrm{sec}$

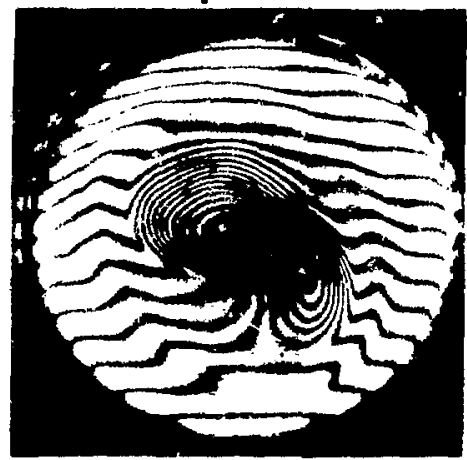

$8.5 \mu \mathrm{sec}$

Fig. I. Time sequence of end-on plasma column interferograms. 


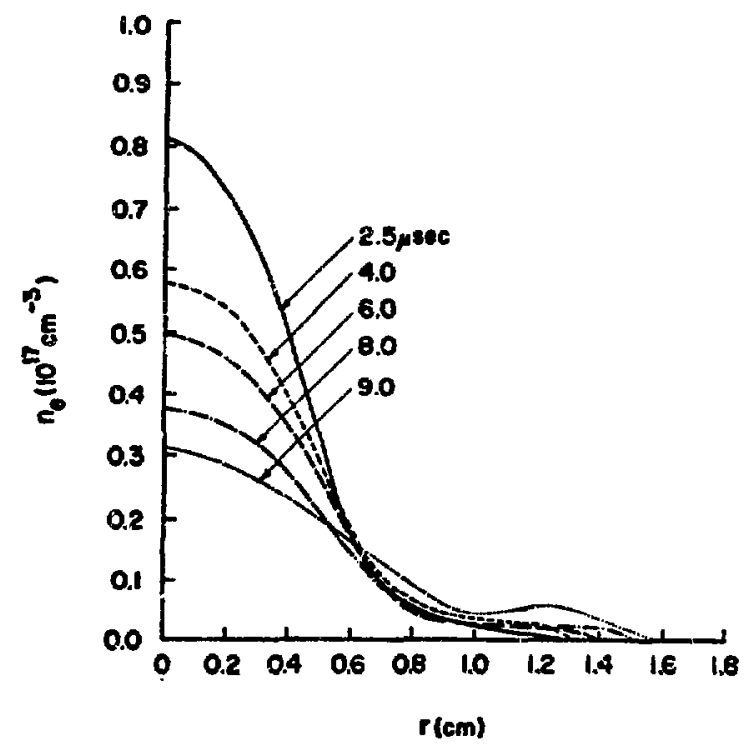

Fig. 2. Plasma electron density profiles for an assumed plasma column length of $100 \mathrm{~cm}$.

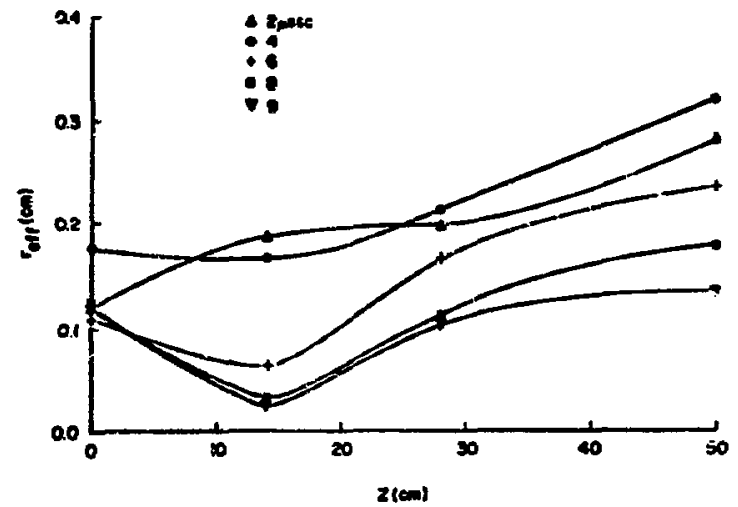

F:g. 3. Axial and temporal evolution of the plasma excluded flux radius.

Four loop-probe systems were arranged along the axis of the discharge tube. With $z=0$ defining one end of the 100-cm long theta-pinch coil, the diamagnetic loopproijes were located at $z=0,14,28$, and $50 \mathrm{~cm}$. The systems were calibrated by discharging the primary bank with a copper rod of known diameter inserted into the discharge tube to simulate the plasma column. With this calibration technique, field iringing effects at the end of the theta-pinch coil $(z=0)$ would not be expected to significantly distort the loop-probe measurement.

The axial time evolution of the effective plasma radius is shown in Fig. 3 . The lines connecting the data points are introduced only as a visual aid since an insufficient number of datz stations are available to precisely define the axial distribution of the excluded flux radius. However, the phenomenology of the time and axially dependent excluded flux radius can be identified. At early times, $t \leqslant 3 \mu s$, the axial distribution of $r_{\text {eff }}$ is as might be expected; i.e., an $r_{\text {eff }}$ minimum at $z=0 \mathrm{~cm}$ and a rise to a maximum value at mid coil $(z=50 \mathrm{~cm})$. The noteworthy feature of Fig. 3 is the gradual decay in $r_{\text {eff }}$ at $z \sim 14 \mathrm{~cm}$. The evolution of the $r_{\text {eff }}(z)$ profiles imply the formation of a magnetic nozzle, or loss orifice, well within the theta-pinch coil. In this case, the temperature and density of the axially flowing plasma would be expected to be a minimum in the nozzle throat region, producing $a$ decrease in $\beta$ and a corresponding decrease in 
excluded flux radius, as observed. The increase in $r_{\text {eff }}$ at $z=0$ could indicate the deceleration ani interaction of the plasma with the discharge tube wall as the plasma expands in the diverging magnetic fields near the ends of the thetapinch coil.

\section{Temperature and B of the Source Plasma}

The excluded flux data, when appropriately combined with the measured plasma density distribution, yield the plasma $\beta .^{6}$ In the high-density plasma column the thermal equilibration time between electrons and ions is small, so that $\left(\mathrm{T}_{\mathrm{e}}+\mathrm{T}_{\mathrm{i}}\right) \approx 2 \mathrm{~T}_{\mathrm{e}}$.

Figure 4 presents the time history of the plasma density, the plasma component temperatures, and the plasma $\beta_{A}$ ( $B$ on axis) determined from the loopprobe data obtained at $\mathrm{z}=50 \mathrm{~cm}$ and the end-on interferograms. Although there is significant scatter in the $\mathrm{T}_{e}=\mathrm{T}_{i}$ and $\mathrm{B}_{\mathrm{A}}$ data points, a reasonable estimate of average values can be made. At peak field the plasma component temperatures have an average value of about $50 \mathrm{eV}$. The temperature slowly decreases to approximately $45 \mathrm{eV}$ at $t \approx 6.5 \mathrm{\mu s}$. After this time a rapid decrease in the plasma temperature is observed with $\mathrm{T}_{\mathrm{e}}=\mathrm{T}_{i} \approx 10 \mathrm{eV}$ just prior to the onset of the destructive $m=2$ instability at $t \approx 9-10 \mu \mathrm{s}$. A similar phenomenon can be $i-$ dentified from the $B_{A}$ data, where $B_{A} \approx 0.44$ for $t \leq 4.5 \mu \mathrm{s}$. For $t>4.5 \mu \mathrm{s}, B_{A}$ decreases rapidly in time to a relatively low value of about 0.05 at $t \approx 9 \mu \mathrm{s}$.

The abrupt decrease in plasma temperature observed at $t \sim 6.5 \mu \mathrm{s}$ could result from the arrival, at the coil midplane, of rarefaction waves which can propagate inward from the ends of the plasma column. Wesson ${ }^{14}$ has derived an expression for the axial velocity of a rarefaction wave traveling in a radially uniform plasma which can be expressed as,

$$
V=\left(\frac{2}{\gamma}\right)^{1 / 2}(1-\beta)^{1 / 2} C_{s} \text {, }
$$

where $\mathrm{C}_{\mathbf{s}}$ is the plasma acoustic velocity. For the parameters of the Scylla I-C plasma, Wesson's model predicts a rarefaction wave arrival time at the coil midplane of $\ell / 2 V \approx 7 \mu \mathrm{s}$, where $l$ is the coil length $(l=100 \mathrm{~cm}$ ) and $B$ has been taken as 0.44 . More recent treatments 18,19 of the plasma end-loss problem, using the MHD approximation and diffuse radial equilibrium, predict the existence of inward traveling rarefaction waves which propagate at the cusp velocity, given by 

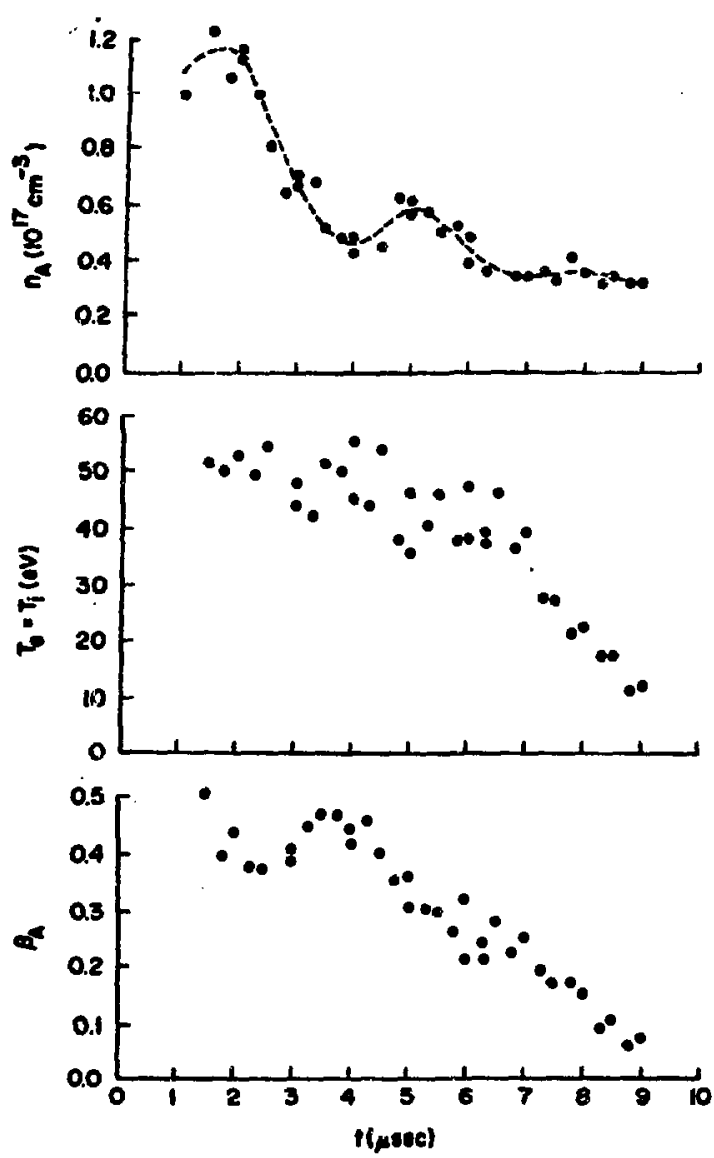

Fig. 4. Time history of the plasme density on axis, the plasma component temperatures, and the plasma beta on axis at

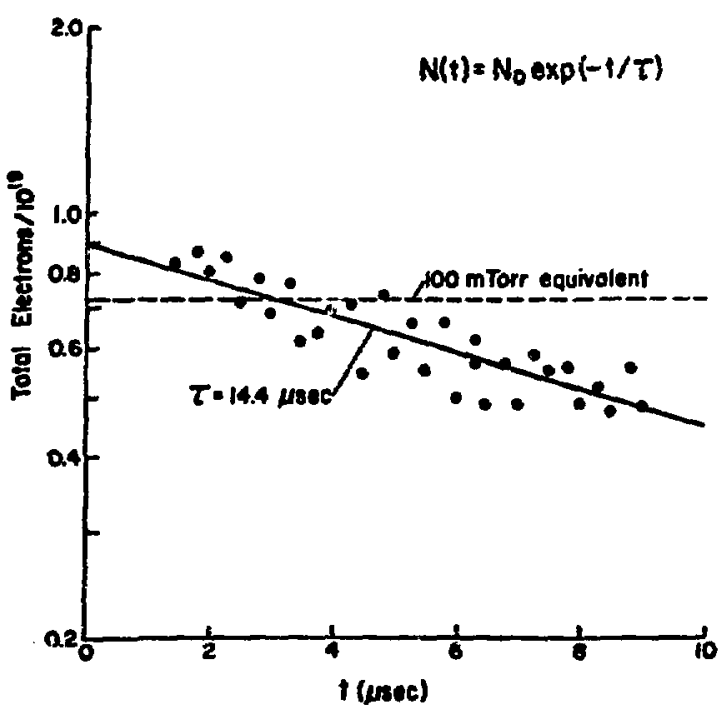
$z=50 \mathrm{~cm}$.

Fig. 5. Time history of the total plasma column electron inventory.

$$
U=\left(\frac{I}{c_{S}^{2}}+\frac{I}{v_{A}^{2}}\right)^{-1 / 2},
$$

where $V_{A}$ is the Alfven speed. Using the cusp velocity, a rarefaction wave is predicted to arrive at the coil midplane at $\ell / 2 U \approx 10 \mu \mathrm{s}$. Although Wesson's model ${ }^{14}$ would appear to agree with the experimental observation, a more detailed experiment in which the axial evolution of the predicted rarefaction waves can be identified is necessary in order to accurately identify the physical characteristics and existence of such waves. 
V. INTERFEROMETRIC DETERMINATION OF PLASMA END-LOSS TIME

The total number of electrons $N_{e}$ contained within the plasma column, at a given time, is determined from the end-on interferograms. ${ }^{6}$ Figure 5 presents the time history of the total electron inventory. At early times, $t<3 \mu \mathrm{s}$, the total number of particles slightly exceeds that expected for the 100-mtorr filling pressure. The difference could result from the introduction, into the plasma, of gas initially adsorbed on the discharge tube walls. Assuming an exponential particle j.nventory decay rate; expressed as

$$
N_{e}(t)=N_{0} \exp (-t / \tau)
$$

when $T$ is defined as the particle end-loss time, a least-squares fit of the data of Fig. 5 yields $\tau=34.4 \mu \mathrm{s}$.

The results of previous end-loss experiments ${ }^{1-3}$ have demonstrated that the end-loss time scales as $l / T_{i}^{1 / 2}$, where $T_{i}$ is the ion temperature. Comparing the end-loss times of those experiments with that of the present experiment an endloss time of $\sim 17 \mu$ s is predicted from the $\ell / T_{i}^{1 / 2}$ scaling law. The Scylla I-C experiment, with $\tau \sim 14 \mathrm{\mu s}$, does not scale directly with the earlier results. In Scylla I-C, where the ion-ion mean free path $\lambda_{j, i} \approx 0.1 \mathrm{~cm}$ is much 1 sss than the dimensions of the column, the plasma is collision dominated whereas in the earlier experiments $^{1-3}$ collisionless plasmas were produced.

Although the difference between the observed Scylla I-C end-loss time ari that expected from the $\ell / \mathrm{T}_{i}^{1 / 2}$ scaling may simply be a result of the large scatter in the data points, and thus the experimental error, it is of interest to compare the particle end-loss rates predicted from elementary theories for the collisional and collisionless regimes with the experimental results. In the collision dominated Scylla I-C plasma column the flow from the ends into the vacuum region should be describable by classical MHD concepts. Teylor and Wesson ${ }^{13}$ have treated the case of steady collisional choked flow through a magnetic nozzle in the presence of internal magnetic fields. The expression they derived for the particle loss rate is

$$
\dot{N}_{s}=2 n_{i} A_{p} C_{s} f(\beta, \gamma)
$$

where $n_{i}$ is the particle number density in the source plasma, $A_{p}$ is the column 12 
cross-sectional area, and $f(\beta, \gamma)$ is an algebraic function of the plasma $\beta$ and the ratio of specific heats. $f(B, \gamma) \approx 0.8$ for the parameters of the Scylla I-C plasma so that Eq. (16) can be approximated as $\dot{i}_{s} \approx 1.6 n_{i} A_{p} c_{s}$. Wesson's ${ }^{14}$ unsteady collisional flow model predicts a particle loss rate given by

$$
\dot{M}_{u}=2 n_{i} A_{p} C_{s} g(\beta, \gamma)
$$

where $g(\beta, \gamma) \approx 0.3$ for $0 \leq \beta \leq 0.8$ and $\gamma=5 / 3$. Thus, in the present experiment $\dot{\mathrm{N}}_{\mathrm{u}}$ can be approximated as $\dot{\mathrm{N}}_{\mathrm{u}} \approx 0.6 \mathrm{n}_{\mathrm{i}} \mathrm{A}_{\mathrm{p}} \mathrm{C}_{\mathrm{s}}$.

In collisionless plasma experinents where the ion-ion mean free path exceeds the dimensions of the plasma column, the plasma particle loss would be expected to be an effusive process rather than a diffusive process encountered in collision dominated plasmes. In the collisionless case the effusive particle loss rate can approximated as

$$
\bar{N}_{c}=-2 A_{0}\left[1 / 4 n_{i} \overline{\bar{c}}\right]
$$

where $\bar{C}=\left(B_{\mathrm{kT}} \mathrm{T}_{\mathrm{i}} / \pi_{\mathrm{m}}\right)^{1 / 2}$ is the mean speed of the ions and $A_{0}$ is the effective area of the plasma column loss orifice. The area of the loss orifice $A_{0}$ can be estimated from the results of the collisionless experiments ${ }^{2-3}$ where the end-loss time, $\tau$, was letermined interferometrically and an exponential decay rate was assumed. Differentiating $\mathrm{Eq} .(15)$ and combining with Eq. (18) and expressing the total number of particles in the plasma colum $\|_{e}$ as $n_{i} A_{p}$ yields

$$
A_{0}=\frac{2 A}{t} \cdot \frac{q}{C}
$$

Fo: the plasm parameters of the three-meter Scylda IV and rive-meter linear Seyllac experiments, it can be stown that Eq. (19) gives $A_{0}=A_{p}$. Thus Eq. (18),

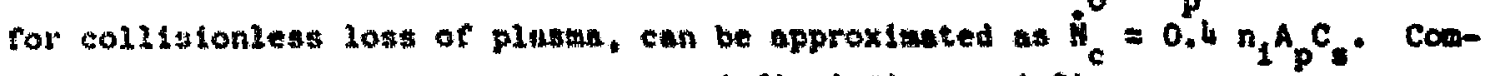
paring the approximated roras of Eq. (16), (17), and (18), the particle end-loss rate rom a collistow dominated pinuma would be expected to exceed that from a

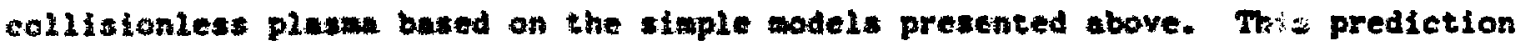

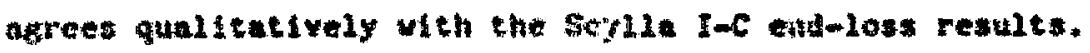




\section{vi. EXAMINATION OF PLASMA END-LOSS WITH A LOCAL PRESSURE MEASURING DIAGNOSTIC}

\section{A. Piezoelectric Pressure Probe}

The development of instrumentation to sense local, gas-kinetic pressure in plasma is an area of continuing interest. Pressure diagnostics, being sensitive to momentum flux, must be in direct contact with the plasma and this presents a unique set of constraints for the instrument. The use of piezoelectric transducers for this application has generally been considered optimum. 20,21 However, such probes are limited in signal/noise ratio, risetime and spatial resolution. Signal/noise problems can be eliminated through use of long acoustical transmission lines between the plasma contact and the piezoelectric crystal. The probe risetime scales as $\mathrm{L}^{1 / 3} \mathrm{e}^{2 / 3}$ where $\mathrm{L}$ is the length and $\mathrm{a}$ is the diameter of the delay line. 22,23 Thus in application a compromise between risetime and signal/ noise ratio is obtained. This type of probe, commonly referred to as a "pressure bar," has been successfully employed in plasma physics research. 24,25

To obtain maximum temporal resolution, pressure probes which do not utilize an acoustic delay line have been constructed. 26 In this configuration the piezoelectric crystal is isolated from the plasma by a thin insulating material. Pressure tranducers of this design have been used in several pulsed plasma experiments $^{27,28}$ including the present Scylla I-C end-loss experiments. The primary disadvantages of probes of this design are the noise pickup problems encountered in high energy plasma discharges and magnetic impact loading of the pressure transducers conducting shell. These problems have been overcome in the present experiment at the expense of the probe risetime.

A schematic of the pressure probe used in the Scylla I-C experiments is shown in Fig. 6. The transducer is protected from the plasma by a cylindrical quartz outer jacket and a $0.25-\mathrm{mm}$ thick quartz disk attached by epoxy to the probe front end. A pressure signal impressed upon the end will cause a stress wave to propagate through the quartz disk to the piezo-ceramic (PZT-5, Clevite Corp., Cleveland, Ohio); the signal delay time through the quartz disk is $\approx 40 \mathrm{~ns}$. The probe was calibrated by using the step pressure increment generated by a reflected shock wave in a simple shock tube. The probe was flush mounted in the shock tube end wall. Figure $6 b$ shows the response of the probe system to the reflected shock. In these calibration tests the probe system included a 15-m length of coaxial cable and a high frequency noise filter with an RC of $150 \mathrm{~ns}$, as used in the theta-pinch experiments. The evident drop in the theoretically constant 
pressure signal between 6 and $12 \mu \mathrm{s}, \mathrm{Fig} .6 \mathrm{~b}$, is not real pressure, and is related to an extraneous effect due to the mounting which held the pressure probe in the shock tube. A probe risetime of approximately 1 us and calibration factor of $0.016 \mathrm{volt} /(\mathrm{std}$.) atm was obtained from the shock tube results.

The probe was mounted through a plate at the end of the theta-pinch discharge tube. Data were recorded with the probe placed at interior axial locations $z=2.5,5.1,7.6,10.2,12.7,15.2$, and $20.3 \mathrm{~cm}$ relative to the coil end $(z=0)$, and at radial positions $R=0,2,4,6,8$, and $10 \mathrm{~mm}$ from the discharge tube axis. Reference data at each axial position were recorded on vacuum shots. During initial experiments, it was found that strong extraneous signals were being induced by magnetic field impact loading of the pressure transducer condicting shell. This effect was alleviated by placing a 2.5-cm long, 3-mm $0 . d$., 6-mm i.d. stainless steel collar around the transducer. The collar was friction fitted over the probe quartz jacket and left the forward edge of the probe unobstructed. Pressure data recorded at $z=2.5 \mathrm{~cm}$ with and without the collar showed no identifiable effect of the collar on the true pressure data history.

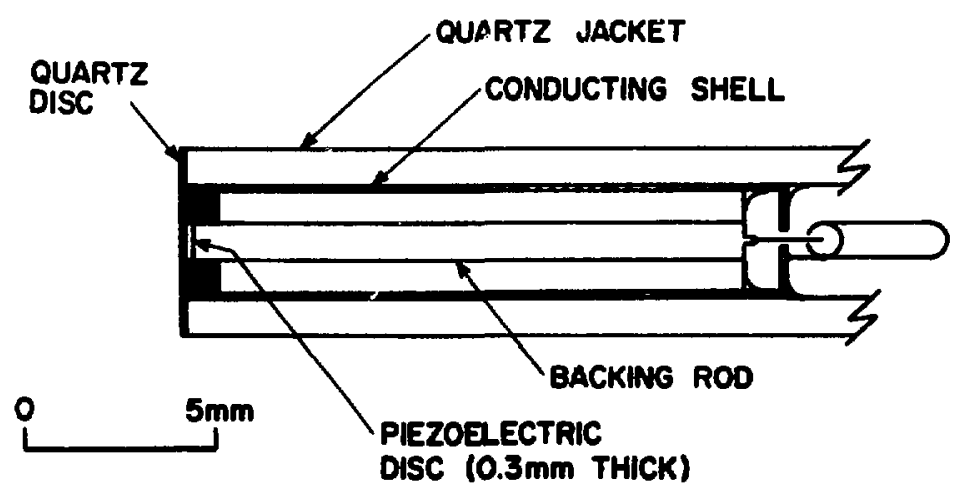

o)

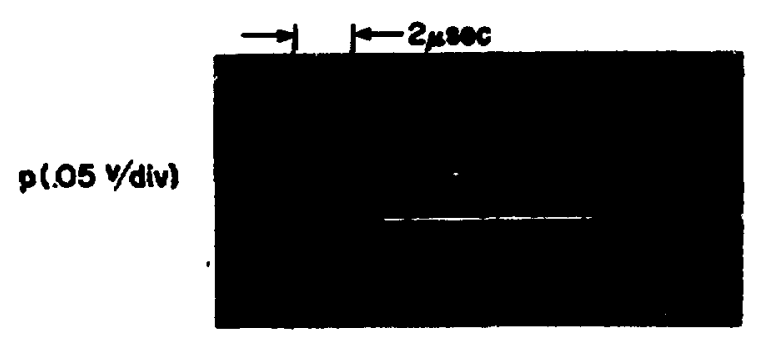

Fig. 6. Schematic of the piezoelectric pressure probe (a), and probe response to a reflected shock wave in the end wall of a shock tube (b).

b) 


\section{B. Plasma Pressure Measurements}

In order to identify any spurious signals introduced into the probe electrical response that are not a direct result of local pinsma pressure, data were recorded under the following conditions: a) vacuum in the discharge tube; $b$ ) 100-mtorr gas fill; c) 100-mtorr gas fill with a quartz disk bonded to the front of the stainless steel collar so that contact between the plasma and the pressure sensitive end of the transducer could not be made. Figure 7 a presents a pressure record (at $\mathrm{R}=0 \mathrm{~cm}, \mathrm{z}=20.3 \mathrm{~cm}$ ) obtained with plasma and $\mathrm{Fig}$. $7 \mathrm{~b}$ shows the corresponding vacuum discharge. The smoothly varying signal resulting from a small amount of residual magnetic loading, Fig. Th, is easily substracted from the pressure signal obtained with plasma,Fig. $\mathrm{Ta}$, in order to determine the true plasma pressure. When contact between the streaming plasma and the pressure sensor is broken (case c), the resulting probe signal is identical to that obtained during a vacuum discharge.

Radial profiles of plasma pressure, reduced from several data secords taken at a given axial and radial position, are presented in Fig. 8 for $z=0$, 7.6 , and $15.2 \mathrm{~cm}$. The pressure profiles at $z=15.2 \mathrm{~cm}$ are nearly Gaussian and decay in magnitude with time, displaying a behavior consistent with axial loss of particles from the plasma column. The profiles obtained at $z=12.7,15.2$, and $20.3 \mathrm{~cm}$ are similar in magnitude and $1 / \mathrm{e}$ radius, and are assumed to be representative of the conditions existing at the coil midplane. The profiles diminish in magnitude and become less Gaussian in shape as the coil end is approached. At $z=0$ the profiles are nearly uniform across the tube radius for times greater than $4.0 \mu \mathrm{s}$, behavior indicative of flowing plasma which has expanded in the diverging magnetic fields near the ends of the theta-pinch coil.

o) PLASima $p(0.1 \% /(w)$

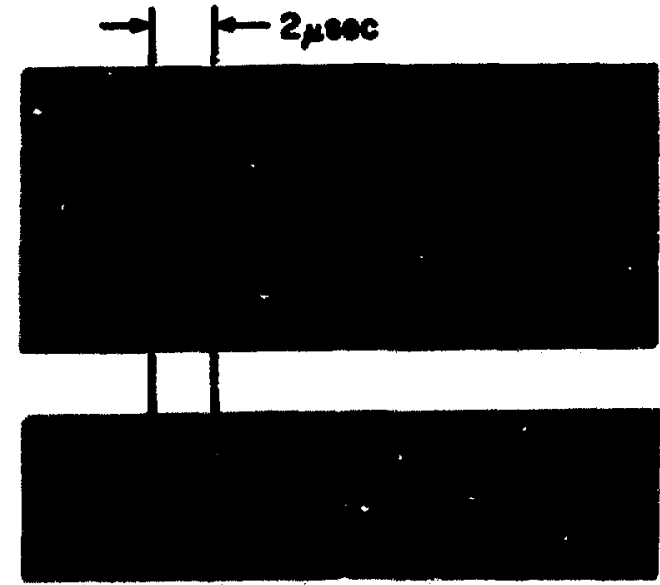

Fig. 7. Pressure probe response in plasma (a), and vacuum (b), discharges. Probe positioned at $z=$ $20.3 \mathrm{~cm}, R=0 \mathrm{~cm}$. 
As previously discussed, the axial evolution of the effective plasma radilis, Fig. 3, obtained from the diamagnetic loop-probe data, indicated the formation of a minimum plasma area of throat region within the theta-pinch coil $(z>0)$. This phenomenon is also observed from examination of the pressure data. Figure 9 presents the $1 / e$ plasma radius, $r_{p}$, obtained from the measured pressure profiles, as a function of axial position and time. For $t \leqslant 6 \mu$ s, a minimum plesma radius of about $0.5 \mathrm{~cm}$ can be identified at $\mathrm{z}=7-10 \mathrm{~cm}$, again well within the theta-pinch coil. At axial positions $z>10 \mathrm{~cm}, \mathrm{r}_{\mathrm{p}}$ approaches the $1 / \mathrm{e}$ plasma radius indicated from the end-on interferograms, $20.6 \mathrm{~cm}$. For $z<7 \mathrm{~cm}$, rapid radial expansion of the plasma is evident. For $t>6 \mu s$, the $r_{p}$ minimum advances further into the coil and a correspondingly greater radial expension is observed.

Defining the minimum plasma area, observed from the pressure data, as the effective particle loss orifice, the magnitude of this orifice can be compared with that predicted from theory. Experimentally the radius of the loss orifice, $r_{0}$, is about $0.5 \mathrm{~cm}$ and the plasma column radius is approximately $0.6 \mathrm{~cm}$ for $t \leqslant 6 \mu \mathrm{s}$. Assuming initially that the radial electric fields in the plasma sheath are not short-circuited, then, as previously discussed, the radius of the loss orifice would be expected to have a maximum value on the order of the geometric
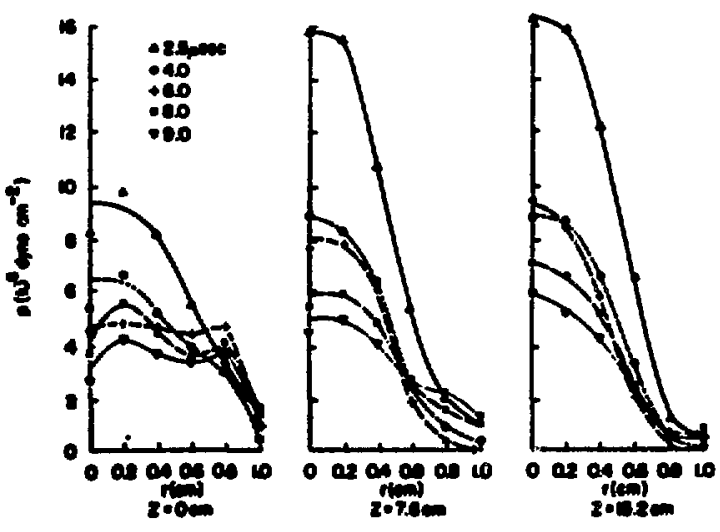

Fig. 8. Radial pressure profiles at $2=0,7.6$, and $15.2 \mathrm{~cm}$.

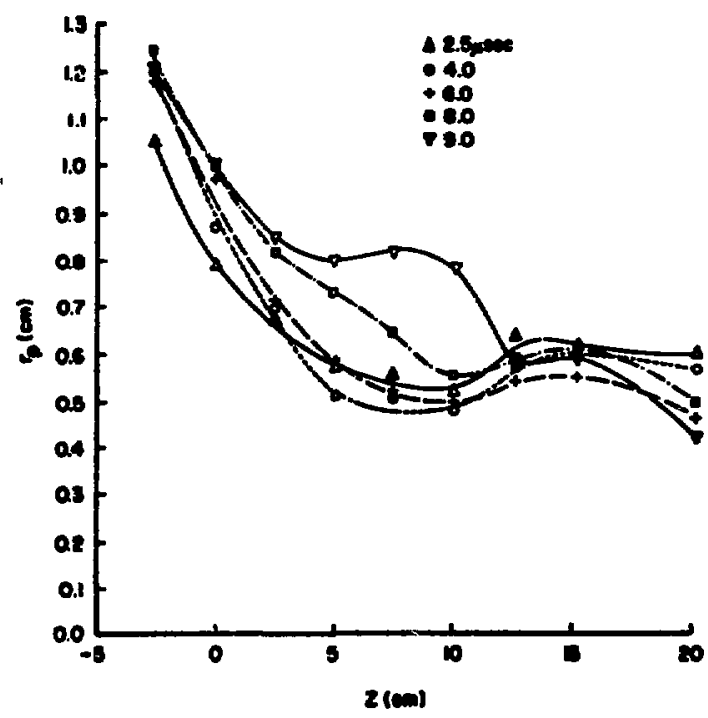

Fig. 9. Axial and temporal variation of the 1/e plasma radius obtained from pressure profiles. 
mean of the electron and ion gryo-radii, $\left(r_{i} r_{e}\right)^{I / 2}$, where $r_{j}=m_{j} v_{o j} c / e B$, end $v_{0}=\left(2 k T_{j} / m_{j}\right)^{l / 2}$ is the particle mean thermal speed. Taking $t=3$ is as a representative time during the loss process when $T_{e}=T_{i} \approx 50 \mathrm{eV}$ and $B=25 \mathrm{kG}$, then

$$
\left(r_{i} r_{e}\right)^{I / 2}=0.007 \mathrm{~cm}
$$

For comparison, the electron gyro-radius $r_{e} \approx 0.001 \mathrm{~cm}$ and the jon gryo-radius $r_{i} \approx 0.06 \mathrm{~cm}$. Assuming that the space charge electric field in the source plasma is short-circuited, then flux corservation in the sheath requires a loss orifice radius on the order of $\sqrt{2}\left(r_{p} r_{i}\right)^{1 / 2}$, Eq. (4). Again taking $t=3 \mu \mathrm{s}$, when $r_{p} \approx 0.6 \mathrm{~cm}$ and $r_{i} \approx 0.06 \mathrm{~cm}$, then

$$
\sqrt{2}\left(r_{p} r_{i}\right)^{1 / 2}=0.27 \mathrm{~cm} \text {. }
$$

The above estimates of the expected loss orifice radius, based on collisionless theories which assume a central core of $B=1$ plasma surrounded by a well defined plasma sheath, predict values of $r_{0}$ which are smaller than that experimentally observed. It is clear from inspection of the density (Fig. 2) and pressure profiles (Fig. 8) that a well defined sheath does not exist in the Scylla I-C plasma column. In addition, $B<1$ and the plasma is collision dominated since the ion-ion mean free path is $\approx 0.1 \mathrm{~cm}$, significantly less than the $1.2-\mathrm{cm}$ plasma diameter. Accordingly, the assumptions on which the collislonless theories are based are clearly violated in Scylla I-C and therefore these theories could not be expected to accurately describe the plasma.

Since the plasma is collisional, the essential physical phenomenon should be describable by MHD theories. The steady MHD plasma loss theory of Taylor and Wesson ${ }^{13}$ predicts an orifice radius equal to $r_{p}(1-\beta)^{1 / 4}, E q$. (5). For the observed plasma parameters at $t \approx 3 \mu \mathrm{s}$,

$$
r_{p}(1-\beta)^{1 / 4}=0.52 \mathrm{~cm}
$$

From We:sson's ${ }^{14}$ treatment of unsteady plasma flow a loss orifice radius of Eq. $16 ;$ 


$$
r_{p}\left(\frac{1-\beta}{\gamma}\right)^{1 / 6}=0.5 \mathrm{~cm}
$$

is indicated for $\gamma=5 / 3$. Thus, the $0.5-\mathrm{cm}$ effective loss orifice radius determined from the pressure data is in excellent agreement with predictions from collisional MHD theories, but in poor agreement with collisionless theories. Based on these results it would appear that theories assuming collisionless plasma behavior cannot be applied in the collisional MHD regime. However, MHD theories have been used in theoretical efforts which attempt to describe the end-loss process in collisionless plesma regimes. The validity of this approach is a fundamental and unanswered plasma physics question.

D. Rate of Plasma Loss

The rate at which particles are lost from one end of the plasma column can be expressed as $J=n A v$, where $n, v$ are number density and flow velocity averaged over the plasma column cross-sectional area, A. The loss rate involves the interaction between the density, temperature, and velocity of the plasma as it varies with time at a given location. Pressure, as it involves a combination of these variables, can be used in conjunction with other diagnostics to indicate the magnitude of plasma loss rate.

The quantity which is sensed by the pressure probe is the momentum flux from the plasma particles impinging upon the probe surface area. The measured pressure $p$, which contains a component due to the random kinetic motion and a component due to directed particle motion, can be approximated as

$$
p=n k\left(T_{e}+T_{1}\right)+n m v^{2}
$$

where $n$ is the local number density, $T$ is the local particile temperature, $m$ is the ion mass and $v$ is the directed plasme velocity. The second term in the above equation assumes a Newtonian interaction of rlowing particles with the probe; the particles do not interact with each other when colliding with the probe surface, but lose their normal monentum component. In the present case where the ion mean free path is of the same order of magnitude as the probs sensing diameter $(1 \mathrm{~mm})$, little upstream influence can be expected and the above formulation provides an adequate description of the plasma-probe interaction. 
In order to determine the component pressures that comprise the total measured pressure, the pressure probe data taken at $\mathrm{z}=15.2 \mathrm{~cm}$ are combined with the diamagnetic loop-probe data obtained at $z=14 \mathrm{~cm}$. It is assumed that strong axial gradients do not exist over this short, $1.2-\mathrm{cm}$, plasma length. Taking the plasma density proriles, determined from the interferograms, to be approximately equal to the density profiles at $z=14 \mathrm{~cm}$, the plasma $\beta$ is evaluated from the loop-probe data obtained at $z=14 \mathrm{~cm}$. The plasma static pressure $\mathrm{p}_{\mathrm{s}}$ is then determined from pressure balance, $p_{S}=n k\left(T_{e}+T_{i}\right)=B B^{2} / 8 \pi$, using the measured value of $B$ and the calculated value of 8 . Then $p-p_{s}=n m v^{2}$ can be evaluated.

While local values of $n(r)$ could lead to local values for flow velocity, $v(r)$, from the pressure data, it is more reasonable to deal with radial average values of $\mathrm{n}$ and $\mathrm{p}$ in order to determine average values of velocity, $\bar{v}$. Since the density and pressure profiles are nearly Gaussian,

$$
\begin{aligned}
& \int_{r=0}^{\infty} n(r) 2 \pi r d r=\int_{r=0}^{\infty} n_{A} e^{-\left(r / r_{p}\right)^{2} 2 \pi r d r=n_{A} \pi r_{p}^{2},} \\
& \int_{r=0}^{\infty} p(r) 2 \pi r d r=\int_{r=0}^{\infty} p_{A} e^{-\left(r / r_{p}\right)^{2} 2 \pi r d r=p_{A} \pi r_{p}^{2},}
\end{aligned}
$$

where $n_{A}$ and $p_{A}$ are the measured density and total pressure on axis. Accordingly, the plasma column is treated as having a uniform density $n_{A}$ and pressure $p_{A}$ over a radius $x_{p}$ (determined from the pressure data). The average flow velocity is then evaluated from $p_{A}-p_{s A}=n_{A} m \vec{v}^{2}$. From this formulation, average flow velocities, $\bar{v}$, slightly less than the ion thermal velocity, $v_{i}$, are indicated for $t<6.0 \mu \mathrm{s}$; at $t=3.0 \mu \mathrm{s}, \bar{v}=7.7 \times 10^{6} \mathrm{~cm} / \mathrm{s}$ and $v_{i}=8.5 \times 10^{6} \mathrm{~cm} / \mathrm{s}$. For times greater than $6 \mu \mathrm{s}, \bar{v}$ becomes somewhat larger than $v_{i}$. It should be noted that this calculation is approximate since $n_{A}$ has been assumed constant in $z$ between the coil midplane and $z=14 \mathrm{~cm}$.

From the calculated values of $\bar{v}$, the total loss rate of particles from both ends of the plasma column can be expressed as

$$
\dot{N}(t)=2 J=2 n_{A}\left(\pi r_{p}^{2}\right) \bar{v}
$$


For comparison, the variation of particle loss rate predicted from the steady collisional theory of Taylur and Wesson, ${ }^{13} \mathrm{Eq} .(16)$, and the unsteady collisional theory of Wesson, ${ }^{14} \mathrm{Eq}$. (17), are evaluated for the time dependent parameters of the present experiment. Figure 10 presents the loss rates determined from the reduction of the end-on interferograms, the pressure probe data, and the steady and unsteady collision dominated flow theories. The two analytical models predict similar functional behavior for loss rates $\left(\dot{\mathrm{N}}_{\mathbf{s}}, \dot{\mathrm{N}}_{\mathbf{u}}\right)$ with time, but there are substantially different loss magnitudes in each case. However, the analytical models and the results of local pressure measurements all indicate an initial period of high particle loss rate ( $t<4 \mu \mathrm{s}$, Fig. 10), which is not resolved by the interferograms. The loss rate determined from the interferograms is based on axial and radial averages of density with an assumed functional form for the particle inventory decay rate and so does not respond to the fine structure of the loss process. The rates obtained from the pressure data tend to follow the high values of the steady theoretical model and also display more detail of the loss process than can be obtained from the interferograms.

\section{DISCUSSION}

The end-loss process in the collision dominated Scylla I-C plasma has been investigated with a local pressure sensitive diagnostic, integrated density measurements and axially arrayed diamagnetic loop probes. The developnent of a plasma loss orifice, well within the theta-pinch coil, has been identified from both local pressure and loop-probe measurements. The magnitude of the observed orifice radius is found to be in excellent agreement with that predicted from collisional MHD theories but in poor agreement with that indicated from collisionless sheath models. The axially flowing plasma is well confined and peaked on axis until it flows through the loss orifice. After passing through the orifice, rapid axial expansion is observed. Over the time scale of the experiment, the axial location of the loss orifice does not significantly change. Inward traveling rarefaction waves were not observed from the loop-probe data; since area changes associated with such waves are significant only at high $\beta$ ( $\beta$ >0.8), such an identification would not be expected at the low $\beta$ values of the present experiment, $\beta \approx 0.44$. However, an indication of the existence of rarefaction waves has been observed from the plasma midplane temperature data. The abrupt decrease in the temperature at $t \approx 6.5 \mu \mathrm{s}$ corresponds to the predicted time of arrival of rarefaction waves at the coil midplane, based on Wesson's transient flow theory. The plasma loss rate derived from the pressure data indicates an initial period 


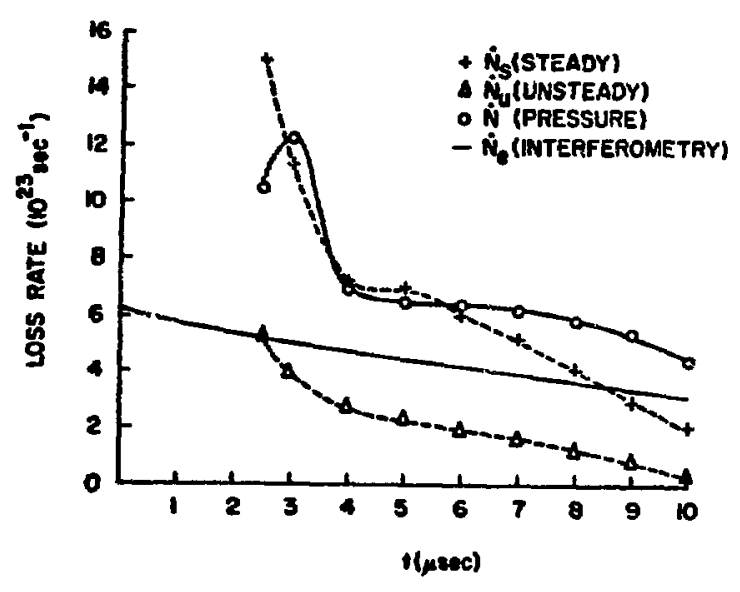

Fig. 10. Comperison of experimentally determined plasma loss rates and loss rates predicted from analytical models.
( $t<4 \mu s)$ of rapid particle loss followed by a period ( $t>4 \mu \mathrm{s}$ ) of gradual decay in the loss rate. This initial period of high loss rate is predicted from the MHD flow theories when the measured time dependent plasma parameters are substituted into the analytical models. The loss rate determined from the end-on interferograms does not respond to the detailed structure of the plasma loss process.

\section{FURTHER CONSIDERATIONS}

The data and analyses discussed above indicate the need for more detailed experimertal and theoretical work on the plasme end-loss problem in both collisional and collisionless regimes. Experimentally, local temperature and density measurements at axial positions common to pressure and diamagnetic loop-probe diagnostics is needed. Since such measurements were not made in the present work, a more detailed analysis of the loss process could not be pursued. For example, there were common loop-probe and pressure data taken at the $z=0$ position, but an analysis of this region assuming an axially independent density distribution, as carried out above, is not relevant because of the large changes in plasma density due to expansion of the flow. Considereble improvement of the loss predictions can be achieved by modeling the plasma iemperature variations, but again, the temperature indication in the present work was indirect and analyses would best be pursued when a temperature sensitive diagnostic is employed. Further, the significance of variables such as sonic, thermal and cusp velocities to the end-loss problem cannot be considered absolute until appropriate analytical models are derived which accurately describe the loss process. Finally, there exists no direct experimental evidence that MHD models, appropriate for collisional plasma, can be used in the description and analysis of collisionless plasma end loss. 


\section{ACKNOWLEDGMENT}

We wish to thank W. T. Armstrong for his aid in the interferometric and loop-probe data reduction, E. I. Zimmermann for maintaining the experimental apparatus and S. E. Linzey for his technical assistance.

\section{REFERENCES}

1. E. M. Little, W. E. Quinn, and G. A. Sawyer, "Plasma End Losses and Heating in the Low Pressure Regime of a Theta Pinch," Phys. Fluids, $\underline{8}, 1168$ (1965).

2. R. F. Gribble, W. E. Quinn, and R. E. Siemon, "Plasma Experiments with a Three-Meter $\theta$ Pinch," Phys. Fluids, 14, 2042 (1971).

3. K. S. Thomas, H. W. Harris, F. C. Jahoda, G. A. Sawyer, and R. E. Siemon, "Plasma Experiments on the Linear Scyllac Theta Pinch," Phys. Fluids, 17, 1314 (1975).

4. J. P. Freidberg and H. Weitzner, "Endloss From a Linear O-Pinch," Nucl. Fusion, 15, 217 (1975).

5. W. R. Ellis, Jr., W. B. Riesenfeld, and G. A. Sawyer, "Proposel for the Construction of a Scylla IV-P Confinement Studies Theta Pinch," Los Alamos Scientific Laboratory Report LA-5474-P, Decenber, 1973.

6. K. F. McKenna, "Plasma Experiments on the Scylla I-C Theta Pinch," Los Alamos Scientific Laboratory Report LA-6397-MS.

7. H. Grad, "Boundary Layer Between a Plasma and a Magnetic Field," Phys. Fluids, $\underline{4}, 1366$ (1961).

8. C. L. Longmire, Elementary Plasma Physics, Interscience Pub., New York, 1963, p. 94.

9. R. L. Morse, "Adiabatic Time Development of Plasma Sheaths," Phys. Fluids, $\underline{8}, 308(1965)$.

10. K. S. Thomas, "Measurement of Shorting Currents from a Theta-Pinch Plasma," Phys. Rev. Lett, 23, $7^{1+6}$ (1969).

11. M. Kaufmann, E. Fiinfer, J. Junker, J. Neuhauser, and V. Seidel, "Rotational Instabilities and Axial Currents in the Theta Pinch ISAR I," Max-PlanckInstitut Für Plasmaphysik, Report IPP 1/123, October 1971.

12. J. Marshall, Los Alamos Scientific Laboratory (private communication).

13. J. B. Taylor and J. A. Wesson, "End Losses from a Theta Pinch," Nucl. Fusion 2, 159 (1965).

14. J. A. Wesson, "Plasma Flow in a Theta-Pinch," in Plasma Physics and Controlled Nuclear Fusion Research (Proc. 2nd Int. Conf. Culham, 1965), I, 223, IAEA, Vienna (1966). 
15. H.A.B. Bodin, J. McCartan, I. K. Pasco, and W. H. Schneider, "Experimental and Two-Dimensional Computational Study of End Losses from a Theta Pinch," Phys. Fluids, 15, 1341 (1972).

16. W. G. Vincenti and C. H. Kruger, Jr., Physical Gas Dynamics, John Wiley and Sons, Inc., New York, 1967, p. 18.

17. C. P. Hobbs and I. J. Spalding, "Some Factors Influencing the Containment of a Cusp Plisma," Culham Laboratory, May, 1964, CLM-R57.

18. J. V. Brackbill, M. T. Menzel, and D. C. Barns, "Numerical Studies of the Linear Theta Pinch, "Third Topical Conference on Pulsed High Beta Plasmas," Culham, England, Sept. 9-12, 1975.

19. H. Weitzner, Courant Institute of Mathematical Sciences, New York University, (private communication).

20. T. G. Jones and G. C. Vlases, "Pressure Probes for Research in Plasma Dynamics and Detonation," Rev. Sci. Insti. 38, 1038 (1967).

21. T. M. York, K. F. McKenna, and C. J. Michels, "Dynamic Pressure Transducer System for Pulsed Plasme Flow Diagnosis," Rev. Sci. Instr., 44 , 588 (1973).

22. I. R. Jones, "A Review of the Pressure Bar Technique for Measuring Transient Pressures," Aerospace Corp. (Los Angeles), Rept. No. TDR-594(120801) TR-3(1961).

23. I. R. Jones, "Beryllium Pressure Bar Having Submicrosecond Risetime," Rev. Sci. Instr. 37, 1059 (1966).

24. H.A.B. Bodin and C. A. Bunting, "A Study of the End Losses from a Theta Pinch Using Piezoelectric Pressure Probes," Culham Laboratory, Jan. 1967, CLM-R70.

25. T. K. Allen, A. J. Cox, and I. J. Spalding, "Cusp Injection Experiment," Plasma Physics and Controlled Nuclear Fusion Research, 2, IAEA, Vienna, 1966 , p. 427.

26. T. M. York, "Stress Dynamics in High Speed Piezoelectric Pressure Probes," Rer. Sci. Instr. 41, 519 (1970).

27. T. M. York and R. G. John, "Pressure Distribution in the Structure of a Propagating Current Sheet," Phys. Fluids, 13, 1303 (1970).

28. K. F. McKenna and T. M. York, "Transient Flow and Expansion of a Pinch Discharge Plasma in Self-Induced Nagnetic Fields," Plasma Phys., 17, 1 (1975). 